\title{
Parameter sensitivity in nonlinear and dissipative time-reversed acoustics
}

\author{
Claes M. Hedberg \\ Department of Mechanical Engineering, University of Karlskrona-Ronneby, Karlskrona, Sweden \\ clacs.hedberg@ima.hk-r.se
}

\begin{abstract}
Time-reversed nonlinear acoustics has shown that a propagated signal retains information about the original signal after shock formation and even after merging of shocks. This information is dependent on the accuracy of the measured or calculated clata. Presented here is a more detailed analysis of the estimation of dimensionless and real parameters in the problem - the original amplitude, the distance traveled and the dissipation over nonlinearity ratio. It is shown that the methods are fairly insensitive to noise.
\end{abstract}

\section{THE METHOD AND ITS PARAMETERS}

Linear time reversed acoustics takes a received signal, and retransmits the time reversed signal in the direction it came from which has applications in medicine, undersea communications, hydrodynamics and material analysis [1]. Linear time reversal takes advantage of time reversal invariance.

Nonlinear time reversal is invariant only when the dissipation is zero and the wave has not been shocked [2]. A wave that has a shock has irretrievably lost some part of its content. Still, if the wave and its derivatives are considered continuous there remain in theory forcver information about the original signal. But factors like numerical accuracy and noise set limits in practical situations.

This article is concerned with nonlinear and dissipative one-dimensional sound waves.

The calculations and results are expressed in dimensionless quantities where $V=\frac{1}{v_{0}}$ is the velocity amplitude, $\sigma=\frac{\omega v_{0} x \beta}{c_{0}^{2}}$ the distance from the source, $\theta=\omega \tau$ the retarded time and $\nu=\frac{b \omega}{2 \beta c_{0} v_{0} \rho_{0}}$ is a ratio between dissipation and nonlinearity. The true quantities are: $v$ is the velocity amplitude, $v_{0}$ is the source velocity amplitude, $\omega$ is the frequency, $x$ is propagated distance, $\beta$ is a nonlinearity measure, $c_{0}$ is the small signal sound speed, $\tau$ is retarded time, $b$ is a dissipation measure and $\rho_{0}$ is the density.

Back-propagation for zero dissipation is made through a simple analytical method where points on the wave are propagated negatively in time $\theta$ in proportion to their amplitude $V$ multiplied by the distance $\sigma$.

For non-zero dissipation the signal is divided into half-periods which are treated locally as sinus waves. For these there exist the Fubini solution Eq.(1) which is good when the distance $\sigma_{\text {sinus }}<1$, and the analytical Fay solution $\mathrm{Eq}_{\mathrm{l}}$.(2) which works well when the distance $\sigma_{\text {sinus }}>3$.

$$
\begin{gathered}
v_{\text {Bessel-Futini }}=2 v_{0} \sum_{n=1}^{\infty} \frac{J_{n}\left(\frac{n \omega v_{0} x \beta}{c_{0}^{2}}\right) \sin (n \omega \tau)}{\frac{n \omega t^{\prime} x \beta}{c_{0}^{2}}} \\
v_{\text {Fay }}=\frac{b \omega}{\beta c_{0} \rho_{0}} \sum_{n=1}^{\infty} \frac{\sin (n \omega \tau)}{\sinh \left[\frac{n b \omega}{2 \beta c_{0} v_{0} \rho_{0}}\left(1+\frac{\omega v_{0} x \beta}{c_{0}^{2}}\right)\right]}
\end{gathered}
$$

The possibility of estimating real parameters from examining a received signal will now be discussed. It is assumed that we know some material parameters such as the soundspeed $c_{0}$ and the density $\rho_{0}$.

For the Fubini solution Eq.(1) this leaves the velocity $v_{0}$ and the distance multiplicd with the nonlincarity $x \beta$. These may be obtained through examining the values of the local amplitude spectra contra the coefficients in $\mathrm{E}_{\mathrm{l}}$ (1). One can not separate $x$ from the non-linearity $\beta$ as they only appear as a product.

The quantities which may be obtained for the Fay solution $E_{1} .(2)$ is the dissipation over nonlinearity ratio $b / \beta$, the velocity $v_{0}$ and the dissipation times the distance $b x$. Another equation is needed and may be found examining a different half-period of the wave with different amplitude where $v_{0}$ is not the same.

We may observe that in the Fay solution $\mathrm{Eq}_{\mathrm{l}}$.(2), the dependence on the source amplitude $v_{0}$ tends to zero when $x \rightarrow \infty$ as $\frac{n b \omega}{2 \beta c_{0} v_{0} \rho_{0}}\left(1+\frac{\omega v_{0} x \beta}{c_{0}^{2}}\right)=\frac{n b_{0}}{2 \beta c_{0} v_{0} \rho_{0}}+\frac{n \omega^{2} b, c}{2 c_{0}^{2} \rho_{i}}$.

Change in parameters is often of practical interest, for example, nonlinearity is in the detection of material fatigue [3], which can be detected for complex and unknown systems using this method. 


\section{RESULTS}

In Figure 1 is an example on the steps of the non-dissipative method. To the right noise has been added. The shocked signal (solid line) has been propagated 46 shock distances (equivalent to $\sigma=5$ ) from a source $V_{0}=-\sin 4 \theta-\sin 5 \theta$ (also solid but larger and non-shocked) The signal is backpropagated the same distance $(\sigma=5)$ and the result is shown (dots). The third curve (dash-dots) is the Fourier series adaption to the rolevant parts of the backpropagated signal. It is seen how this result is decent on the left and how naturally added noise, on the right, makes the result worse.
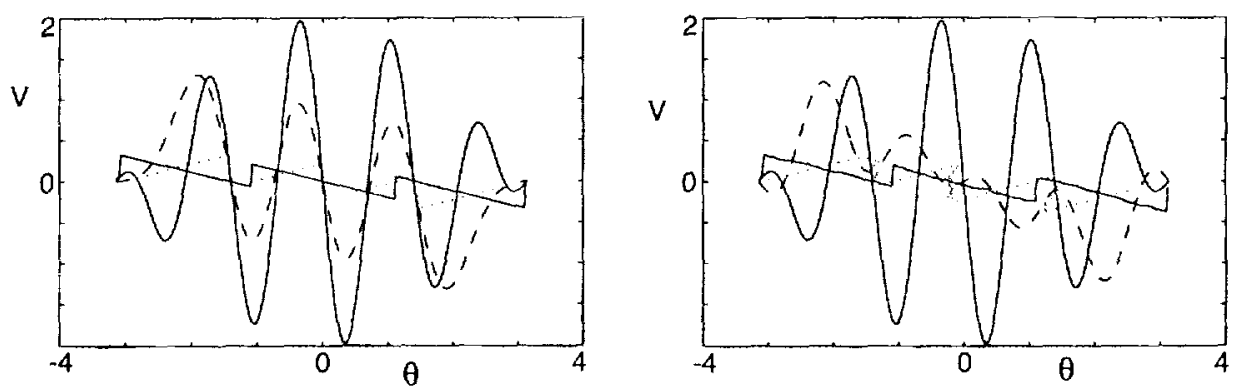

FIGURE 1. Non-dissipative results from $\sigma=5$ for $V_{0}=-\sin 4 \theta-\sin 5 \theta$; noise on the riglit.

Except for the added equation mentioned in the previous section, multi-frequency waves are in general more easily to determine due to the increased input information. In Figure 2 a sinus wave repeated over 5 cycles has been propagated 5 shock distances $(\sigma=1)$ in a dissipative medium $(\nu=0.05)$. On the top row left the signal is pure and on the right some noise has been added. The results after back-propagation (dashed) and the original source signal (solid) are seen below.
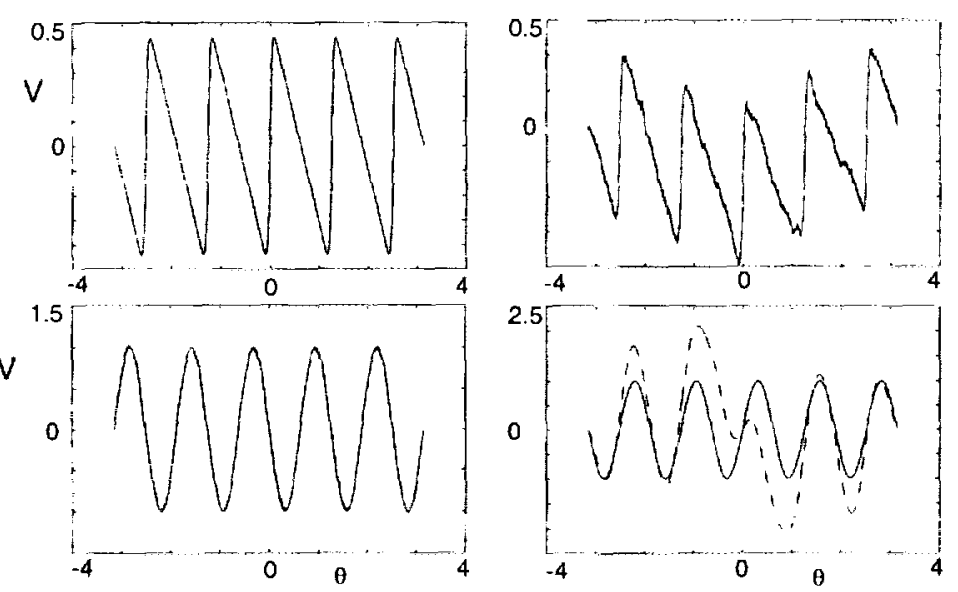

FIGURE 2. Dissipative $\nu=0.05$ at $\sigma=1$ for $V_{0}=\sin 5 \theta$; noise on the right.

It has been shown how and when it is possible to use nonlinear backpropagation to estimate the propagation distance, the source amplitude and the dissipation over nonlinearity ratio. From these the source signal may be recreated.

\section{REFERENCES}

1. Fink, M., Physics Tuday, March (1997)

2. Hedberg, C.M., submitted December 3, 1997 to J.Acoust.Soc.Am.

3. Sutin, A., "Nonlinear acoustic non-destructive testing of cracks" Nonlinear Aconstics in Perspective, Proceedings of the 14th International Symposium on Nonlinear Acoustics, Nanjing, China, 70-74, 1996. 